\title{
Patrones sinópticos aplicados a la predicción en la península antártica
}

https://doi.org/10.31978/639-19-010-0.163

\author{
Francisco Vasallo López ${ }^{1}$ (fvasallol@aemet.es) \\ Sergi González Herrero' ${ }^{1}$ (sgonzalezh@aemet.es) \\ Francisco Javier Sanz de las Heras'1 (fsanzd@aemet.es) \\ Benito Elvira Montejo ${ }^{1}$ (belviram@aemet.es) \\ Jaime Fernández García-Moya ${ }^{1}$ (jfernandezg@aemet.es) \\ Jesús Riesco Martín1 (jriescom@aemet.es)
}

${ }^{1}$ AEMET / Grupo antártico

\begin{abstract}
RESUMEN
Aplicando el método de análisis mediante clústeres al campo de presión en superficie de los reanálisis del modelo ERA Interim, se han definido cinco patrones para el área del paso de Drake y la península antártica. El análisis de frecuencias muestra que los cinco presentan una ocurrencia anual similar pero una gran variabilidad estacional, y la persistencia de cada uno es relativamente alta. La transición entre patrones diferentes tiende a seguir un ciclo acorde con el desplazamiento de un cuarto de onda hacia el este de las ondas sinópticas.

El estudio de las configuraciones típicas de la región, basado en los patrones obtenidos, y su relación con el tiempo asociado en superficie (en particular, efemérides) en las bases antárticas españolas (BAE) favorecerá la comprensión de la meteorología de la zona. Además, la aplicación de estos patrones a situaciones cotidianas facilitará y hará más eficiente el trabajo diario de predicción operativa en las campañas antárticas españolas.
\end{abstract}

PALABRAS CLAVE: patrones de presión; clústeres; climatología sinóptica; península antártica; meteorología antártica.

\section{INTRODUCCIÓN}

El uso de clústeres en meteorología permite clasificar de manera objetiva las situaciones sinópticas sin alcanzar un alto grado de abstracción y constituye una metodología práctica e intuitiva para relacionar la meteorología con otras ciencias, cuyas investigaciones dependen del comportamiento de la atmósfera en una determinada región.

Diversos autores han realizado varios intentos de clasificación de las situaciones sinópticas en el sector de la península antártica (KeJNA, 1993; GOVORUKHA y TIMOFEYEV, 2002; TURNER et al., 1998), pero solo hay un estudio previo basado en clústeres (COHEN et al., 2013), aunque referido a la región del mar de Ross, al oeste de esta zona. Una clasificación de este tipo puede conformar una buena base sobre la que estructurar la predicción operativa en una región poco conocida, como es la Antártida. 


\section{EL CONJUNTO DE PATRONES}

Gracias al análisis mediante clústeres del campo de presión a las 12 UTC obtenido del modelo ERA-Interim para el periodo comprendido entre 1979 y 2016, se han identificado (GonZÁLEZ et al., 2018) cinco patrones sinópticos característicos (figura 1) de la zona que comprende la península antártica y el paso de Drake $\left(45^{\circ}-75^{\circ} \mathrm{S} ; 20^{\circ}-120^{\circ} \mathrm{W}\right)$. Cada uno de ellos se ha caracterizado y descrito después de analizar su desarrollo y evolución, y denominado de acuerdo a sus características más destacadas:

- LWS: baja sobre el mar de Weddell (Low over the Weddell Sea).

- LAB: baja sobre los mares de Amundsen y Bellingshausen (Low over the Amundsen and Bellingshausen Seas).

- LDP: baja sobre el paso de Drake (Low over the Drake Passage).

- ZDP: flujo zonal sobre el paso de Drake (Zonal over the Drake Passage).

- RAP: dorsal sobre la península antártica (Ridge over the Antarctic Peninsula).
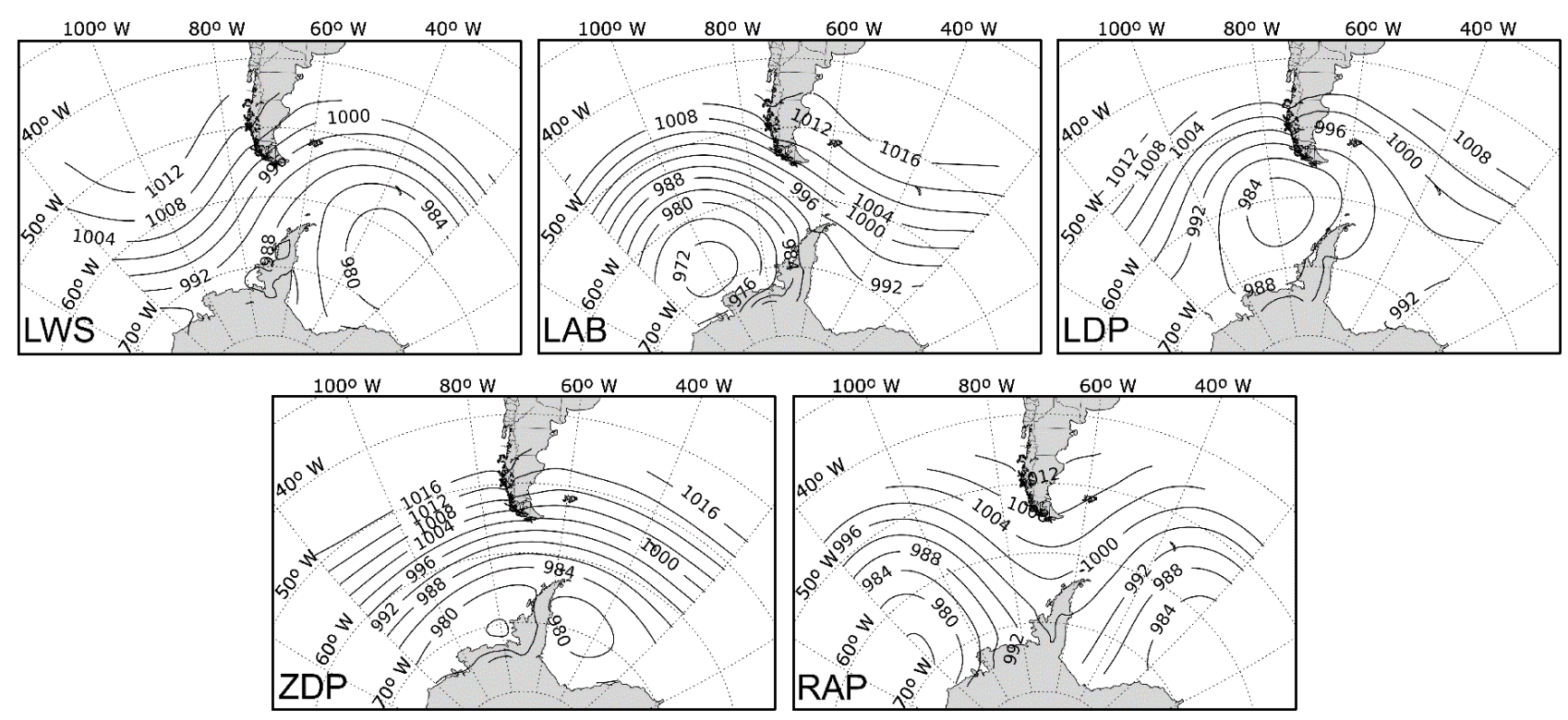

Figura 1. Conjunto de patrones sinópticos obtenidos a partir de análisis del modelo ERA-Interim para el área de la península antártica (GoNZÁLEZ et al., 2018).

Los cinco patrones presentan una frecuencia anual similar, pero una notable variación estacional (tabla 1a). La persistencia de cada uno (repetición de un mismo clúster en días consecutivos) es relativamente alta (tabla 1b), ya que se presenta en dos de cada tres días, indicando que el periodo de desplazamiento característico de los elementos que configuran cada clúster es superior a un día. Los valores de la diagonal muestran la persistencia diaria de cada uno (GonZÁLEZ et al., 2018).

a)

\begin{tabular}{|l|lllll|}
\cline { 2 - 6 } \multicolumn{1}{c|}{} & LWS & LAB & LDP & ZDP & RAP \\
\hline DJF & 16,5 & 16,0 & 28,9 & 21,4 & 17,1 \\
MAM & 17,4 & 20,6 & 20,8 & 19,4 & 21,8 \\
JJA & 23,0 & 19,3 & 17,5 & 19,5 & 20,6 \\
SON & 16,5 & 26,0 & 13,7 & 27,8 & 15,9 \\
Total & 18,4 & 20,5 & 20,2 & 22,0 & 18,9 \\
\hline
\end{tabular}

b)

\begin{tabular}{c|c|ccccc|}
\cline { 3 - 7 } \multicolumn{1}{c|}{} & & LWS & LAB & LDP & ZDP & RAP \\
\hline \multirow{4}{*}{ patrón } & LWS & 12,6 & 0,0 & 0,9 & 1,8 & 3,1 \\
& LAB & 0,0 & 13,8 & 2,7 & 3,1 & 0,9 \\
& LDP & 2,6 & 1,0 & 12,9 & 2,0 & 1,8 \\
& ZDP & 2,8 & 1,8 & 1,5 & 14,1 & 1,9 \\
RAP & 0,4 & 3,9 & 2,2 & 1,0 & 11,3
\end{tabular}

Tabla 1. a) Frecuencia anual y estacional (\%) de cada patrón sinóptico.

b) Frecuencia $(\%)$ de una determinada secuencia de patrones en dos días consecutivos. 
GonZÁLEZ et al. (2018) analizaron las transiciones y comprobaron que la transición entre patrones diferentes tiende a seguir un ciclo acorde con el desplazamiento de un cuarto de onda hacia el este de las ondas sinópticas (figura 2). Aunque la sucesión de patrones cambia de una estación a otra, tiende a predominar este ciclo.

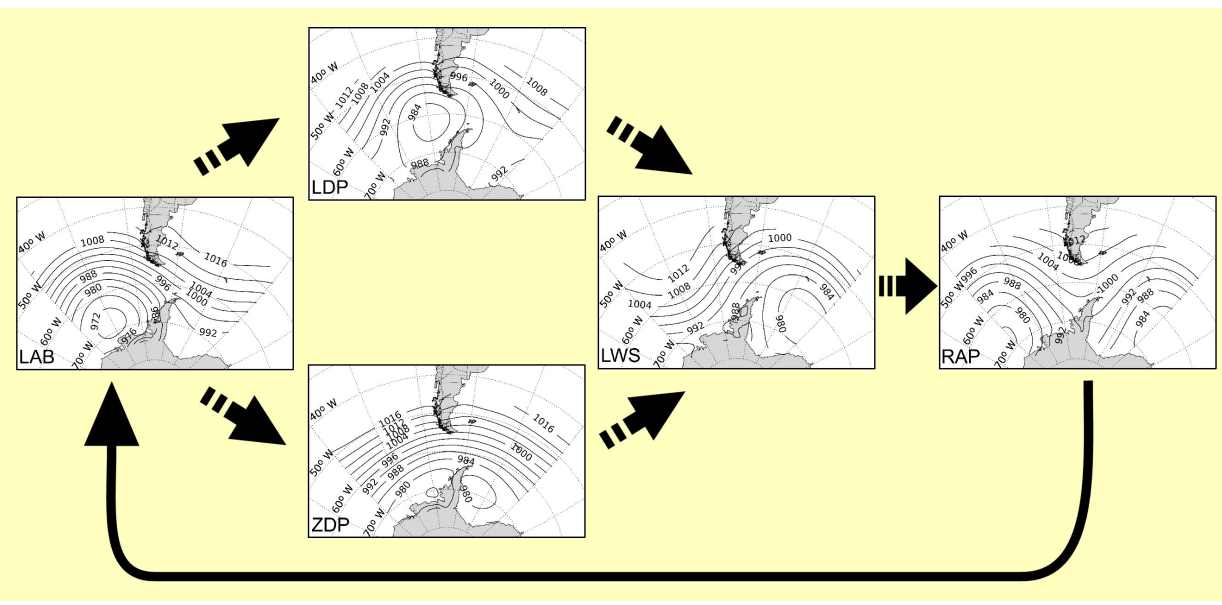

Figura 2.

Transiciones más

frecuentes entre patrones sinópticos en la península antártica.

Todos los patrones sinópticos muestran una mayor correlación con el SAM (Southern Annular Mode, Modo Anular del Sur) que con el ENSO (El Niño-Southern Oscillation, El Niño-Oscilación del Sur). Durante la fase positiva del SAM, la depresión de los mares de Amundsen y Bellingshausen (ABSL) se ve reforzada y la circulación se vuelve más zonal (TURNER et al., 2013; RAPHAEL et al., 2016; Clem y FogT, 2013), favoreciendo la ocurrencia de ZDP y LAB y reduciendo la de LDP y LWS. Por contra, durante la fase negativa predominan las depresiones sobre el paso de Drake y el mar de Weddell.

\section{INFLUENCIA DE LOS CLÚSTERES SOBRE LA METEOROLOGÍA DE LAS BASES ANTÁRTICAS ESPAÑOLAS}

Las islas Shetland del Sur, donde se encuentran las BAE, se sitúan prácticamente en el centro de la zona de estudio elegida por GonZÁLEZ et al. (2018), por lo que es de esperar que las masas de aire que llegan a la zona estén relacionadas con el patrón circulatorio imperante. Se ha demostrado que las anomalías de temperatura y humedad diarias en la estación automática de la base Juan Carlos I (JCI) en la isla Livingston, con respecto al valor medio mensual, varían con la situación sinóptica.

Por ejemplo, se puede apreciar que LAB favorece la entrada de aire cálido y húmedo del Pacífico sureste, mientras que LWS y RAP advectan aire continental frío y seco. Tanto ZDP como LDP exhiben temperaturas templadas, pero mientras ZDP transporta aire húmedo sobre todo, LDP presenta un comportamiento doble, ya que muestra dos máximos de la función de densidad con diferente contenido de humedad. Este comportamiento puede deberse a la diferente advección de aire en función de la posición de la depresión respecto a la isla. Cuando la depresión está situada al oeste de Livingston se advecta aire cálido y húmedo, y cuando esta se desplaza al este el aire se vuelve más frío y seco.

Las anomalías de precipitación diaria con respecto a la media mensual en la estación automática de la BAE JCI también cambian para cada patrón circulatorio. Los días con LAB presentan mayores anomalías positivas de precipitación. Los días con ZDP muestran en general anomalías positivas aunque también presentan muchos días con anomalía negativa.

La mayoría de días secos están clasificados como RAP, LWS o LDP. La dualidad que presenta LDP para la humedad también se evidencia para la precipitación, con dos máximos relativos de la función de densidad tanto para anomalías positivas como negativas de precipitación. De hecho, las mayores anomalías de precipitación en JCI no se deben a LAB, sino más bien a los patrones LDP y ZDP; esto indica que no están asociados a LAB los episodios de precipitación intensa, sino más bien los de llovizna débil. 


\section{DESCRIPCIÓN DE LOS PATRONES Y CARACTERÍSTICAS DEL TIEMPO ASOCIADO A CADA UNO}

\subsection{LAB (Depresión sobre los mares de Amundsen y Bellingshausen)}

Patrón circulatorio marcado por la semipermanente ABSL, coincidiendo la posición de la depresión que la caracteriza con la de la ABSL en verano (TURNER et al., 2013; FogT et al., 2012; Hosking et al., 2013). Más que por depresiones móviles, las situaciones incluidas en este patrón están caracterizadas por amplias depresiones cuasiestacionarias, frecuentemente con otras secundarias más rápidas en torno a la principal. Puede tener como contrapartida al otro lado del paso de Drake el anticiclón subtropical atlántico, en forma de cuña o núcleo secundario.

Es característico el flujo cálido y húmedo de norte o noroeste sobre el paso de Drake y la península antártica, cuya frecuencia tiene gran peso en la evolución de las temperaturas en la propia península y en la mitad occidental del mar de Weddell (TURNER et al., 2013; Hosking et al., 2013). Con frecuencia este flujo se concreta en un episodio de «río atmosférico», siendo este grupo el que presenta una mayor correlación positiva con este tipo de eventos. Es el caso de la situación representada en la figura 3, en que las isobaras presentan una configuración típica de LAB, y en la distribución de temperaturas destaca la entrada de aire cálido sobre las islas Shetland del Sur y el extremo norte de la península antártica.

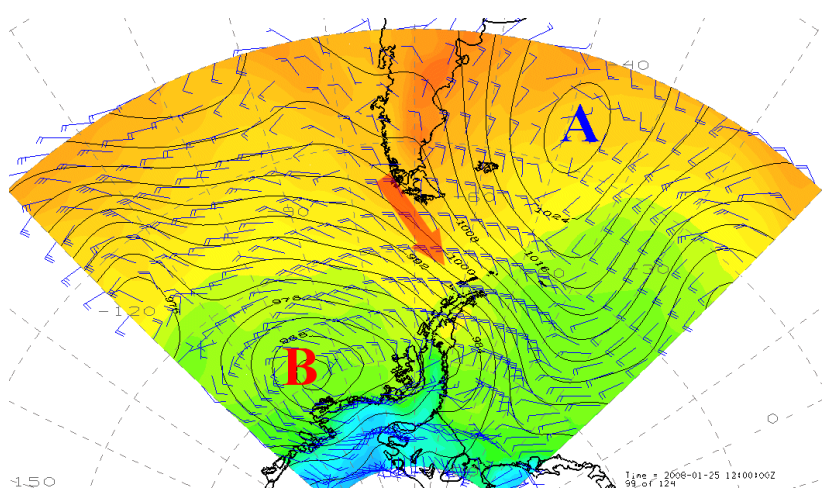

Figura 3. Campos de presión en superficie, temperatura a 2 metros y viento a 10 metros (análisis de ERA-Interim) para

el 25 de enero de 2008 a las 12 UTC.

Los frentes suelen llegar ocluidos a las Shetland del Sur, con precipitaciones persistentes aunque en general poco intensas, y que pueden ser en forma líquida durante el verano. En el sur del paso de Drake son importantes los temporales del cuarto cuadrante, acompañados de condiciones de mar adversas debido a que la persistencia y/o el fetch suelen tener valores elevados. Otros fenómenos característicos de este grupo son las brumas, nieblas y nubes bajas.

La configuración de la figura 4 no es tan típica de LAB como la de la figura 3 , pero presenta las características de precipitación del grupo y un flujo intenso del cuarto cuadrante sobre el paso de Drake, con temporal de NW en las BAE: $22,1 \mathrm{~m} / \mathrm{s}$ de media y 28,6 de racha en JCI y $18,5 \mathrm{~m} / \mathrm{s}$ de media y 27,1 de racha en Gabriel de Castilla (GdC).

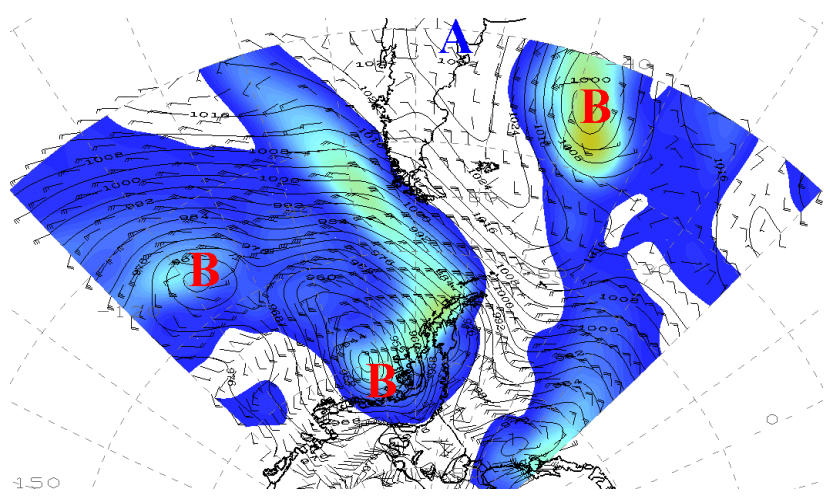

Figura 4. Presión en superficie, viento a 10 metros y precipitación acumulada en las 12 horas anteriores (análisis de ERA-Interim) del 23 de septiembre de 2016 a las 12 UTC.

\subsection{LDP (Depresión sobre el paso de Drake)}

Representa a las depresiones que se sitúan sobre el paso de Drake o al oeste del mismo, siguiendo una trayectoria tanto zonal como meridiana, y que lo atraviesan o quedan bloqueadas en su movimiento en dirección este, siendo desviadas en este caso hacia el norte o el sur. Las situaciones de bloqueo pueden asociarse a la cuña anticiclónica insinuada sobre el Atlántico sur, capaz de conectar con el anticiclón continental antártico, en tanto que el anticiclón subtropical del Pacífico se ve desplazado hacia el oeste. 


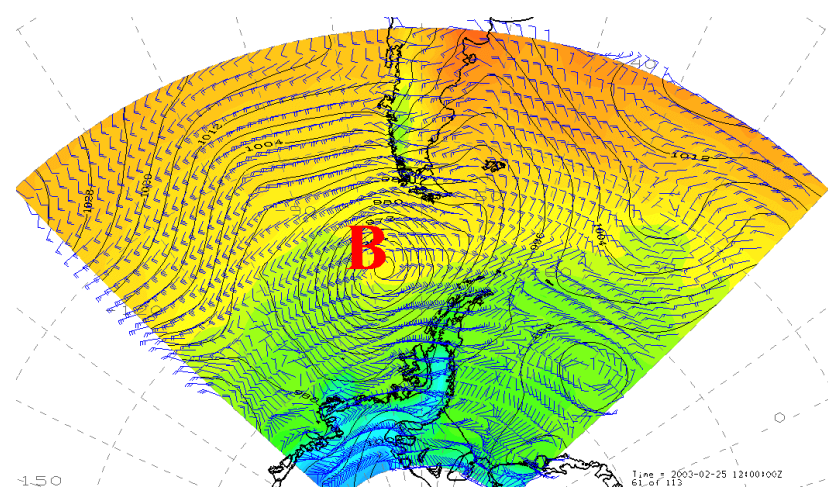

Figura 5. Presión en superficie, temperatura a 2 metros y viento a 10 metros (análisis de ERA-Interim) del 25 de febrero de 2003 a las 12 UTC.

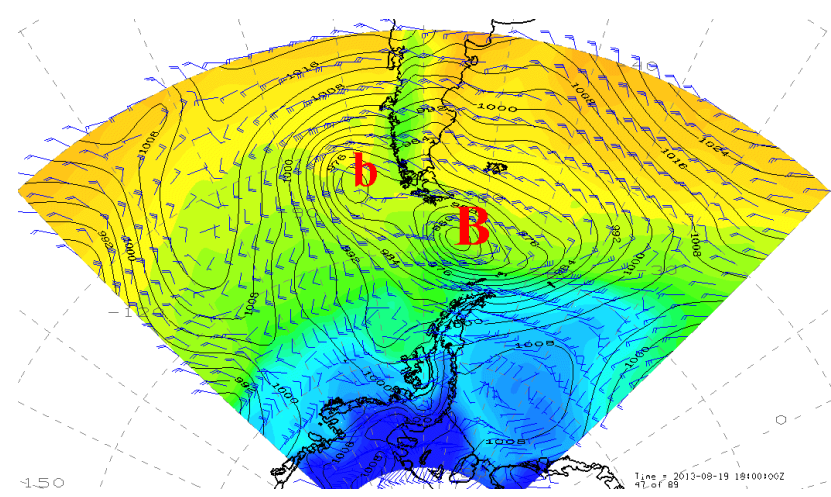

Figura 6. Presión en superficie, temperatura a

2 metros y viento a 10 metros (análisis de ERA-Interim) del 19 de agosto de 2013 a las 18 UTC.

Este grupo se caracteriza sobre todo por depresiones móviles, lo que puede dar lugar a variedad en la dirección del flujo, en función de la posición respecto de la baja, aunque en la zona de estudio predominan los vientos comprendidos entre N y SE. En las BAE son característicos los temporales de ENE con estas situaciones, en las que suelen producirse las precipitaciones de mayor intensidad, y en segundo lugar los de SE, sobre todo cuando el centro de la baja se encuentra al norte de las Shetland del Sur, transitando el paso de Drake de oeste a este. El estado de la mar, aunque desfavorable, puede no serlo tanto como pueda indicar la intensidad del viento, por poca persistencia o escaso fetch.

En la zona de las BAE, las precipitaciones con LDP suelen ser más intensas que con $\mathrm{LAB}$, pero menos persistentes y más irregulares. El 25 de febrero de 2003 (figura 5) una depresión queda parcialmente bloqueada por una cuña al norte del mar de Weddell, mientras el frente asociado se desplaza lentamente hacia las Shetland del Sur hasta quedar casi estacionario sobre las islas. Tras casi doce horas de precipitaciones continuas, se produce el mayor registro en la Base JCI, 53,4 mm en 24 horas, con viento de $\mathrm{E}$ a NE que alcanzó rachas de $25 \mathrm{~m} / \mathrm{s}$.

Cuando una depresión atraviesa el paso de Drake de oeste a este (transición de LDP a LWS) las mayores intensidades de viento en las BAE se alcanzan en plena transición, cuando la depresión se encuentra al norte de las Shetland del Sur. Durante la tarde del 19 de agosto de 2013 (figura 6) se produce uno de los temporales más intensos que se han registrado en la BAE JCI, con vientos de SE reforzados por efecto orográfico (posible viento de ladera descendente) que alcanzan $26,2 \mathrm{~m} / \mathrm{s}$ de media de $140^{\circ}$ y $50 \mathrm{~m} / \mathrm{s} \mathrm{de}$ racha. Se puede comprobar también como en una misma situación puede producirse simultáneamente viento fuerte de componente oeste en el norte del paso de Drake y de componente este en el sur.

\subsection{ZDP (Circulación zonal sobre el paso de Drake)}

Caracteriza a este patrón el intenso flujo zonal de poniente sobre el paso de Drake (figura7), y los mínimos de presión a ambos lados de la península antártica representan a las depresiones móviles insertas en la vaguada circumpolar que se desplazan zonalmente desde el mar de Bellingshausen al de Weddell, sufriendo un proceso orográfico más o menos complejo al atravesar la península antártica (MAYES, 1985).

Suelen predominar las depresiones poco extensas, aunque profundas, y de rápido movimiento. La alternativa la constituyen las depresiones extensas, generalmente con varios centros, que se estacionan sobre la península antártica o se desplazan lentamente al este, y que producen un amplio flujo ciclónico: zonal en el paso de Drake y Cono Sur de Sudamérica, y que se curva al oeste y este de la zona de estudio. Pueden presentarse temporales de W, NW o SW, y tanto la persistencia y como el fetch pueden alcanzar valores altos y la altura del oleaje aumentar notablemente. Las precipitaciones suelen ser débiles en las Shetland del Sur con este patrón. 


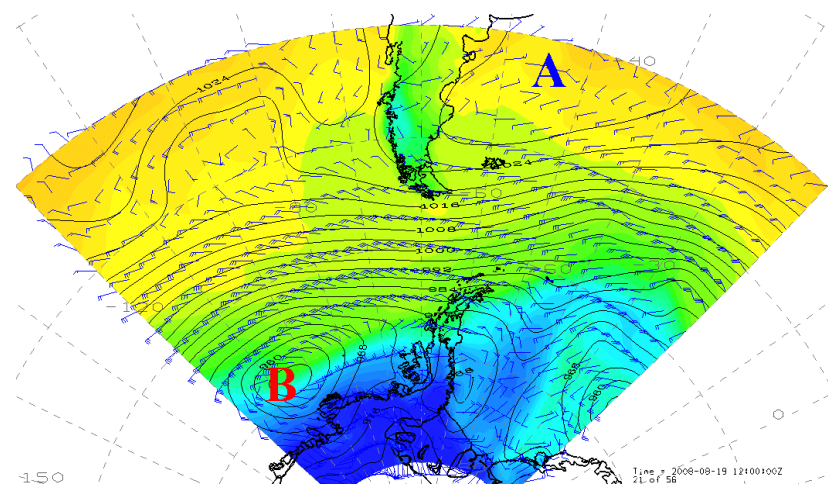

Figura 7. Presión en superficie, temperatura a 2 metros y viento a 10 metros (análisis de ERA-Interim) del 19 de agosto de 2008 a las 12 UTC.

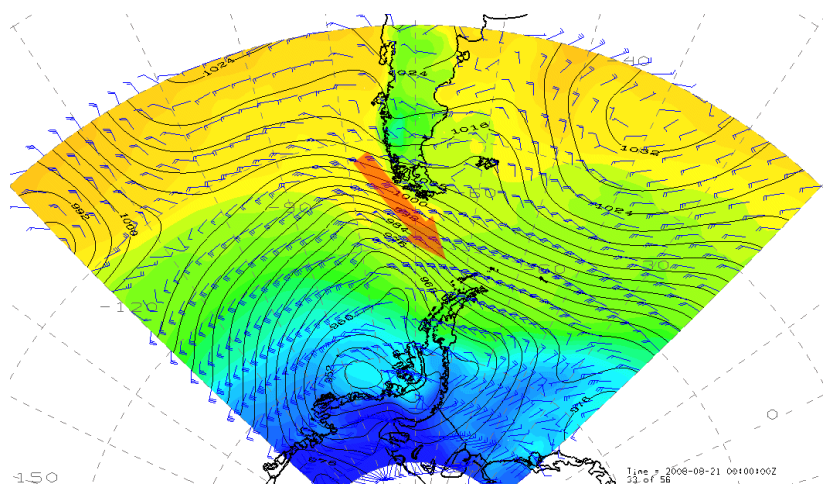

Figura 8. Presión en superficie, temperatura a 2 metros y viento a 10 metros (análisis de ERA-Interim) del 21 de agosto de 2008 a las 00 UTC.

En general se alternan advecciones cálidas y frías más o menos intensas, pero siempre húmedas, y de origen marino. La invasión de aire cálido durante los días 20 y 21 de agosto de 2008 (figura 8) puede compararse a las entradas de $\mathrm{NW}$ propias del patrón $\mathrm{LAB}$, y mantiene las temperaturas en las $\mathrm{BAE}$ por encima de $0{ }^{\circ} \mathrm{C}$ durante más de 24 horas en invierno.

Es típico de este patrón el paso rápido de depresiones del mar de Bellingshausen al de Weddell, atravesando la península antártica y sufriendo efectos orográficos acompañados de procesos de ciclogénesis.

\subsection{LWS (Depresión sobre el mar de de Weddell)}

Este grupo refleja la presencia del anticiclón subtropical del Pacífico sur, que se manifiesta bien como núcleo secundario independiente o bien como una cuña que se extiende hacia el mar de Bellingshausen, conectando incluso con el anticiclón continental antártico. Al otro lado del paso de Drake una depresión al este del extremo de la península antártica representa tanto el máximo de ciclogénesis situado sobre esta zona y el mar de Weddell (Simmonds y KeAY, 2000; Simmonds et al., 2003), como las depresiones que sobrepasan el paso de Drake procedentes del oeste, e incluso las que se forman a sotavento de la cordillera de los Andes y se desplazan en dirección sureste.

Las depresiones resultantes de los procesos de ciclogénesis en el mar de Weddell no suelen afectar a las $\mathrm{BAE}$, ya que en general el desplazamiento predominante las aleja de las mismas, en cambio, las que transitan el paso de Drake de oeste a este sí pueden producir vientos fuertes y precipitaciones. Este tránsito suele llevar asociada una transición de LDP a LWS, y un cambio de viento del primer al segundo cuadrante.

Aunque con este patrón es compatible una variedad de flujos, debido a que las depresiones que lo configuran suelen ser móviles, sobre el paso de Drake, la península antártica y las Shetland del Sur los más característicos e interesantes son los de componente sur, como se puede ver en la situación del 14 de mayo de 2002 (figura 9), que está caracterizada por una profunda y extensa depresión al este de la península antártica. En la vertiente oriental de esta, sobre el mar de Weddell, predomina el flujo de S a SE, mientras que en la vertiente occidental y en el paso de Drake predomina el de SW. Con situaciones de este tipo se pueden producir temporales del tercer cuadrante.

Las masas de aire así advectadas sobre el área del paso de Drake y las Shetland del Sur suelen ser frías y secas, por lo que, salvo de las depresiones que atraviesan el paso de Drake de oeste a este, poca precipitación o ninguna podemos esperar de estas situaciones debido al poco contenido de humedad de las masas de aire continentales movilizadas, pero por el hecho de transitar del suelo helado al mar relativamente más cálido, pueden dar lugar a procesos de ciclogénesis mesoescalar (mesobajas o bajas polares). 


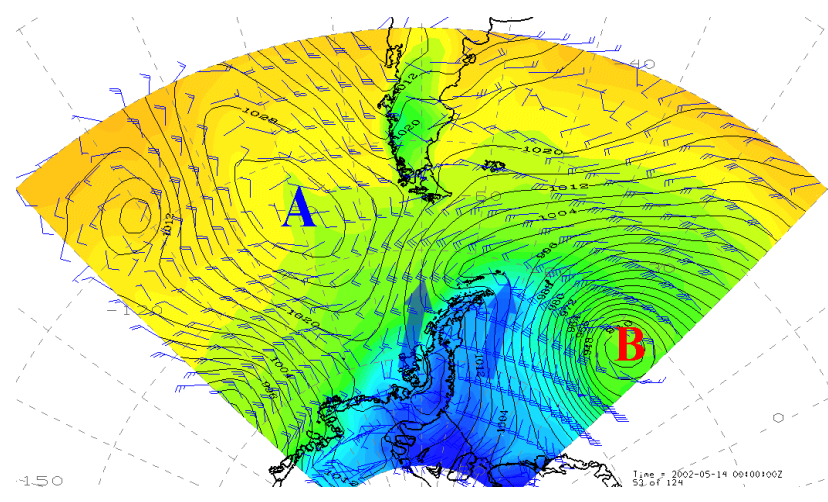

Figura 9. Presión en superficie, temperatura a 2 metros y viento a 10 metros (análisis de ERA-Interim) para el 14 de mayo de 2002 a las 00 UTC.

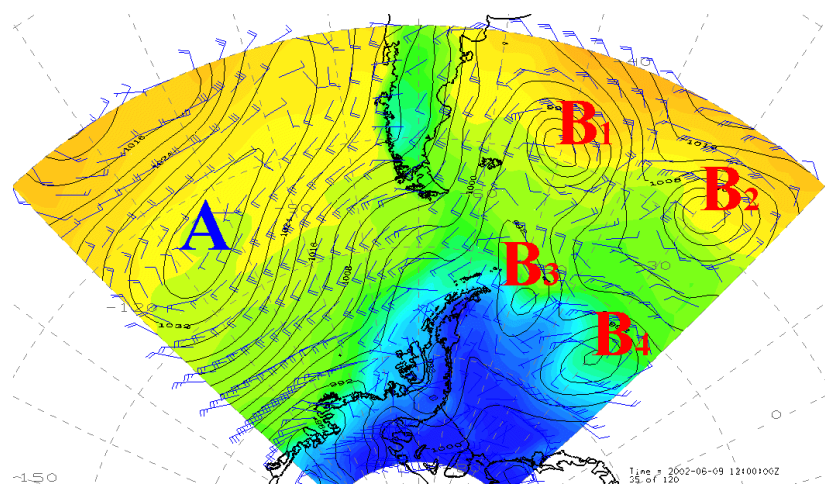

Figura 10. Presión en superficie, temperatura a 2 metros y viento a 10 metros (análisis de ERA-Interim) para el 9 de junio de 2002 a las 12 UTC.

El aire es mucho más frío al este de la península antártica, como se ve en la fig. 9, sobre el helado mar de Weddell, y cuando estas masas de aire alcanzan las Shetland del Sur podemos esperar una ola de frío en las BAE. Pero si el flujo de aire de S o SE sufre el efecto orográfico de la península antártica puede hacer subir sensiblemente las temperaturas, alcanzándose temperaturas superiores a $\operatorname{los} 0^{\circ} \mathrm{C}$ en invierno, o a $10^{\circ} \mathrm{C}$ en verano.

Debido a los variados procesos de ciclogénesis y a las rutas de desplazamiento de las depresiones compatibles con este patrón, las configuraciones englobadas en este grupo pueden estar caracterizadas por depresiones poco extensas y móviles. En este sentido, es interesante la situación del 9 de junio de 2002 (figura 10), donde se pueden ver hasta cuatro vórtices ciclónicos al este del paso de Drake, de los cuales dos (B1 y B2) se han originado a sotavento de los Andes, el tercero (B3) mediante un proceso de ciclogénesis en el extremo de la península antártica, y el último (B4) ha atravesado de oeste a este el paso de Drake. A pesar de la complejidad de la configuración, el flujo sobre la península antártica y el paso de Drake es de componente S, y la masa de aire advectada, fría y seca.

\subsection{RAP (Cuña anticiclónica sobre la península antártica)}

Caracterizado por una cuña anticiclónica que se extiende con una marcada orientación longitudinal desde el Cono Sur de Sudamérica a la Antártida occidental, coincidiendo con la dorsal situada sobre el sur de Sudamérica en el patrón circulatorio de número de onda 3 (VAn LoOn y JenNe, 1972; IRVING y SimMONDS, 2015). Flanqueando la cuña por el este y el oeste, sendas depresiones, extensas y poco móviles, completan este patrón de flujo preferentemente meridiano.

En los ejemplos más típicos de este patrón sinóptico predomina la circulación meridiana, con el eje de la dorsal situado sobre la zona de Cono Sur-paso de Drake-península antártica, llevando asociado un máximo de presión en la misma (figura 11). En otros casos frecuentes el eje puede estar desplazado al este o al oeste, y a veces el anticiclón puede bascular hacia el norte o hacia el sur, diversificando el tipo de situaciones, aunque predominan las advecciones frías y secas al este de la península antártica y cálidas y húmedas al oeste. Las precipitaciones más abundantes se producirán en la vertiente oeste de esta, sobre todo en su mitad sur, que es una conocida zona de máximo de precipitación, en tanto que en las Shetland del Sur son poco importantes, y estas situaciones se saldan generalmente solo con nubes bajas abundantes.

La situación del 2 de septiembre de 2015 (figura 12) es más zonal de lo acostumbrado en este grupo y, al estar situada la dorsal al oeste de la península antártica, el flujo de aire continental frío y seco alcanza su vertiente occidental. Lleva aparejado un temporal de SW sobre el paso de Drake y las Shetland del Sur, que se hace notar en $\mathrm{GdC}$, con 26,8 m/s de media y 33,6 de racha de $240^{\circ}$ el día 1 —en situación ZDP-y 26,5 de media y 31,9 de racha de $235^{\circ}$ el día 2 . 


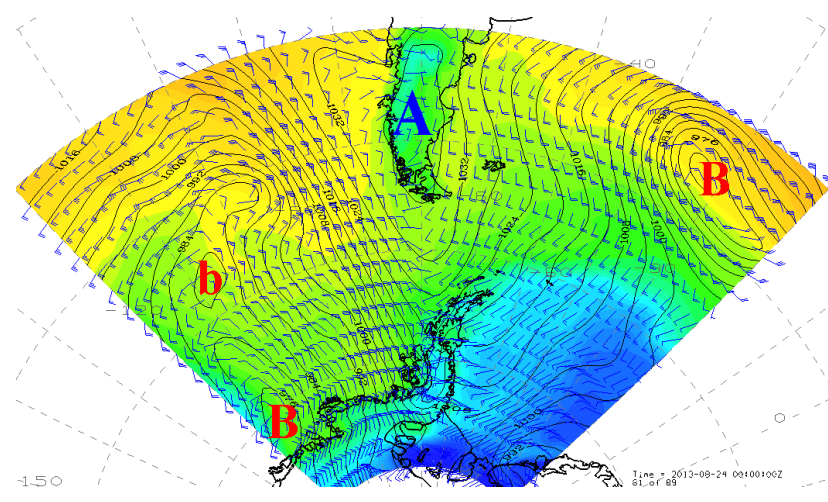

Figura 11. Presión en superficie, temperatura a 2 metros y viento a 10 metros (análisis de ERA-Interim) del 24 de agosto de 2013 a las 00 UTC.

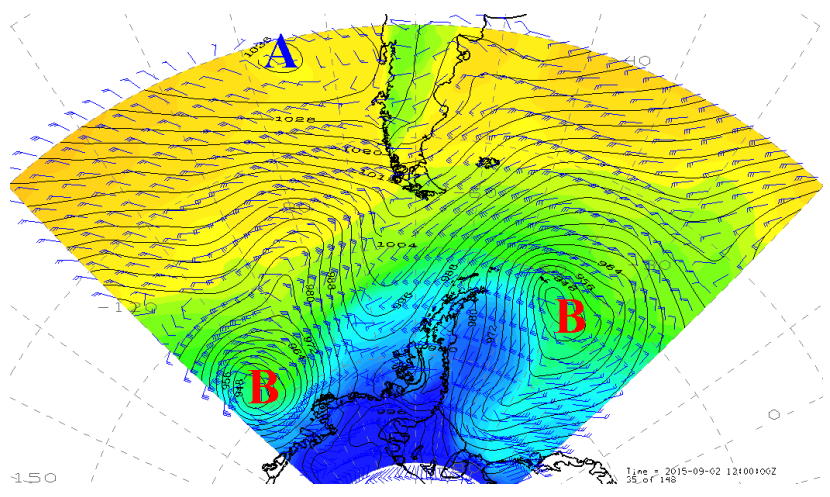

Figura 12. Presión en superficie, temperatura a 2 metros y viento a 10 metros (análisis de ERA-Interim) del 2 de septiembre de 2015 a las 12 UTC.

\section{CONCLUSIONES}

El conjunto de patrones resume — hasta donde conocemos de la meteorología antártica— bastante bien la variedad de situaciones sinópticas en la zona de estudio.

La clasificación objetiva mediante clústeres es una base sólida sobre la que asentar una climatología sinóptica de este sector de la Antártida.

La climatología sinóptica supone un notable avance en el conocimiento de la meteorología de esta región, lo que puede facilitar el avance de otras disciplinas cuyas investigaciones dependen del comportamiento de la atmósfera.

El estudio y la clasificación de situaciones reales de acuerdo con el conjunto de patrones constituye una herramienta útil para adquirir conocimiento y transmitir experiencia en la predicción operativa en la Antártida, sobre todo para predictores que no se dedican a ello de manera continuada.

\section{AGRADECIMIENTOS}

Se utilizó el software Integrated Data Viewer (IDV) de UCAR/Unidata en el análisis y visualización de los datos.

\section{REFERENCIAS}

Clem, K. R. y R. L. Fogt, 2013. Varying roles of ENSO and SAM on the Antarctic Peninsula climate in austral spring. J. Geophys. Res. Atmos., 118, 11481-11492. https://doi.org/10.1002/jgrd.50860.

Cohen, L., S. Dean y J. Renwick, 2013. Synoptic weather types for the Ross Sea region, Antarctica. J. Climate, 26, 636-649. https://doi.org/10.1175/JCLI-D-11-00690.1.

Fogt, R. L., A. J. Wovrosh, R. A. LAngen e I. Simmonds, 2012. The characteristic variability and connection to the underlying synoptic activity of the Amundsen-Bellingshausen Seas low. J. Geophys. Res., 117, D07111. https://doi.org/10.1029/2011JD017337. 
González, S., F. Vasallo, C. Recio-Blitz, J. A. Guijarro y J. Riesco, 2018. Atmospheric Patterns over the Antarctic Peninsula. J. Climate, 31, 3597-3608. https://doi.org/10.1175/JCLI-D-17-0598.1.

Govorukha, L. S. y V. Y. Timofeyev, 2002. Synoptic circulation types of Antarctic Peninsula and adjacent South Ocean regions and connected phenomena. Probl. Klimatol. Polarn., 10, 159-178.

Hosking, J. S., A. Orr, G. J. Marshall, J. Turner y T. Phillips, 2013. The influence of the AmundsenBellingshausen Seas low on the climate of West Antarctica and its representation in coupled climate model simulations. J. Climate, 26, 6633-6648. https://doi.org/10.1175/JCLI-D-12-00813.1.

IRVING, D. e I. Simmonds, 2015. A novel approach to diagnosing Southern Hemisphere planetary wave activity and its influence on regional climate variability. J. Climate, 28, 9041-9057. https://doi.org/10.1175/ JCLI-D-15-0287.1.

KeJnA, M., 1993. Types of atmospheric circulation in the region of H. Arctowski Station (South Shetland Islands) in the years 1986-1989. Proc. XX Polar Symp., Lublin, Poland, Polish Academy of Sciences, 369-378.

MAYES, P. R., 1985. Secular variations in cyclone frequencies near the Drake Passage, southwest Atlantic. J. Geophys. Res., 90, 5829-5839. https://doi.org/10.1029/JD090iD03p05829.

RAPHAEL, M. N. et al., 2016. The Amundsen Sea low: Variability, change, and impact on Antarctic climate. Bull.Amer. Meteor. Soc., 97, 111-121. https://doi.org/10.1175/BAMS-D-14-00018.1.

Simmonds, I. y K. Keay, 2000. Mean Southern Hemisphere extratropical cyclone behavior in the 40-year NCEP-NCAR reanalysis. J. Climate, 13, 873-885. https://doi.org/10.1175/1520-0442(2000)013<0873: MSHECB>2.0.CO;2.

Simmonds, I., K. KeAY y E.-P. Lim, 2003. Synoptic activity in the seas around Antarctica. Mon. Wea. Rev., 131, 272-288. https://doi.org/10.1175/1520-0493(2003)131,0272:SAITSA.2.0.CO;2.

Turner, J., G. J. Marshall y T. A. Lachlan-Cope, 1998. Analysis of synoptic-scale low pressure systems within the Antarctic Peninsula sector of the circumpolar trough. Int. J. Climatol., 18, 253-280. https://doi.org/ 10.1002/(SICI)1097-0088(19980315)18:3<253::AID-JOC248>3.0.CO;2-3.

Turner, J., T. Phillips, J. S. Hosking, G. J. Marshall y A. Orr, 2013. The Amundsen Sea low. Int. J. Climatol., 33, 1818-1829. https://doi.org/10.1002/joc.3558. 
